\title{
Globe
}

Revue internationale d'études québécoises

\section{La question de la souveraineté. Redéfinition des enjeux et nouveaux argumentaires}

\section{Jacques Beauchemin}

Volume 1, numéro 1, 1998

Raisons communes

URI : https://id.erudit.org/iderudit/1000101ar

DOI : https://doi.org/10.7202/1000101ar

Aller au sommaire du numéro

Éditeur(s)

Globe, Revue internationale d'études québécoises

ISSN

1481-5869 (imprimé)

1923-8231 (numérique)

Découvrir la revue

Citer cet article

Beauchemin, J. (1998). La question de la souveraineté. Redéfinition des enjeux et nouveaux argumentaires. Globe, 1(1), 53-75.

https://doi.org/10.7202/1000101ar
Résumé de l'article

Le résultat du référendum de 1995 a eu pour effet de révéler le clivage qui semble s'être creusé entre ce qu'il faut bien appeler le Québec francophone et ses autres, les Québec anglophone et allophone. Cet article porte sur la redéfinition des enjeux soulevés par ce clivage politique et par l'apparition d'un nouvel argumentaire qui, s'appuyant sur la primauté du droit et de la démocratie, structure maintenant le débat autour d’une éventuelle accession du Québec à la souveraineté. Nous tentons de mettre à jour les implications stratégiques de ces incantations au droit et à la démocratie mais, plus fondamentalement, nous cherchons à montrer comment ces arguments nouveaux désignent le lieu d'un problème véritable : celui de la faisabilité et de la légitimité de l'entreprise. La victoire d'un oui signifierait celle d'une majorité de francophones sur les minorités anglophone et allophones. Cela ne veut pas dire qu'une proclamation de souveraineté consécutive à une victoire référendaire serait illégitime, mais qu'un tel clivage signale à tout le moins les difficultés auxquelles se heurterait le nationalisme francophone quant à la mise en oeuvre concrète d'une éventuelle souveraineté.
Tous droits réservés @ Globe, Revue internationale d’études québécoises, 1998 services d'Érudit (y compris la reproduction) est assujettie à sa politique d'utilisation que vous pouvez consulter en ligne.

https://apropos.erudit.org/fr/usagers/politique-dutilisation/ 


\title{
La question de la souveraineté. Redéfinition des enjeux et nouveaux argumentaires
}

\author{
Jacques Beauchemin \\ Université du Québec à Montréal
}

\section{La souveraineté dans une société divisée}

Le résultat du référendum de $1995 \mathrm{a}$ eu pour effet de révéler au grand jour le clivage qui semble s'être creusé entre ce qu'il faut bien appeler le Québec francophone et son autre, le Québec anglophone et allophone. Certes, une part importante de francophones, environ $40 \%$, se représente tout autant comme Québécois que comme Canadien et s'est rangée aux côtés des tenants du «non». Il n'en demeure pas moins que la répartition du vote référendaire, en épousant la ligne de partage linguistique, a considérablement avivé le différend qui oppose les protagonistes en présence. La situation actuelle, en posant clairement face-à-face les groupes en fonction du clivage Québécois francophones "de souche», d'une part, et, d'autre part, Québécois anglophones "de souche» et allophones ayant dans la plupart des cas adopté l'anglais comme langue d'usage, cette situation nouvelle donc, vient préciser les termes du contentieux. En effet, le débat entourant le référendum de 1995 a posé la question de la souveraineté dans le cadre d'une opposition entre droits collectifs et droits individuels alors que le référendum de 1980 avait été l'occasion d'une vaste discussion portant, suivant que l'on était souverainiste ou fédéraliste, sur les avantages ou les périls économiques du projet. Le

Jacques Beauchemin, «a question de la souveraineté. Redéfinition des enjeux et nouveaux agumentaires", Globe. Revwe intermationale d'fivdes quebtcoises, vol. 1, no 1, 1998. 
déplacement relativement récent du centre de gravité du débat tient à un ensemble de déterminations relevant des formes nouvelles de la régulation sociale dans les sociétés capitalistes avancées et de la conjoncture intemationale. Pour aller à l'essentiel, on peut dire que les questions du droit et de la démocratie occupent une position prééminente dans le discours politique contemporain en raison de ce que l'on peut qualifier de judiciarisation de la régulation des rapports sociaux (les cours de justice tendant à se substituer au législatif, ou , si l'on veut, le droit tendant à se substituer au politique) et de la formidable revalorisation de l'idéal démocratique à la faveur de l'effrondrement des «socialismes réels»".

Je m'intéresse dans les pages qui suivent à la redéfinition des enjeux soulevés à la fois par la mise à jour du clivage politique que je viens d'évoquer et par l'apparition d'un nouvel argumentaire qui, s'appuyant sur la primauté du droit et de la démocratie, structure maintenant le débat autour d'une éventuelle accession du Québec à la souvemineté. Je tente de mettre à jour les implications stratégiques de ces incantations au droit et à la démocratie mais, plus fondamentalement, je cherche à montrer qu'au-delà de leurs portée stratégique, ces arguments nouveaux désignent le lieu d'un problème véritable : celui de la faisabilité et de la légitimité de l'entreprise. Bien sûr, le droit à l'autodétermination ne saumit être nié en aucune façon aux Québécois. Mais le projet souverainiste n'en est pas moins confronté aux obstacles que dresse devant lui la très forte opposition qu'il suscite et qui menace la réalisation concrète d'une souveraineté qu'un vote référendaire favorable aurait avalisée. Voilà qui soulève en même temps l'épineuse question de la légitimité que l'on manipule, il est vrai, avec un certain opportunisme. Mais ces manipulations ne soustraient pas les

1 Voir Jacques Beauchemin, «La question nationale québécoise : les nouveaux paramètres de l'analysen, Recherches sociographiques, vol. XXXIX, nos 2 et 3, printemps 1998. 
souverainistes à l'obligation de prendre au sérieux les objections qui surgissent naturellement dans la perspective d'une très courte victoire lors d'un éventuel troisième référendum².

La mise à jour des nouveaux enjeux et des argumentaires en présence exige tout d'abord de revenir sur les racines du contentieux dans le cadre de la dynamique politique canadienne. On aperceva alors plus clairement l'infléchissement du débat actuel.

\section{Le nationalisme québécois dans la dynamique politique canadienne}

Considérée de l'étranger, la crise «nationalitaire» qui semble constituer l'ordinaire de la dynamique politique canadienne étonne d'autant que l'on partage un peu partout la vision euphorique du premier ministre canadien selon laquelle le pays qu'il dirige serait le umeilleur au monde». Monsieur Chrétien répercute en cela les données d'une avantageuse analyse qui situe régulièrement le Canada au premier rang du classement mondial des Nations unies effectuée sur la base de l'indice $d u$ développement humain. Pourtant, ce grand pays semble incapable de satisfaire les aspirations politiques de l'une de ses principales constituantes, au point où cette demière menace de faire sécession.

2 J'écris ces lignes au lendemain de la tenue du congrès national du Parti Québécois en septembre 1998. Le premier ministre Bouchard est parvenu à repousser l'éventualité d'un nouveau référendum en posant l'exigence que ce demier ne soit tenu que lorsqu'auront été réunies les «conditions d'un référendum gagnant». Faut-il préciser que le contentieux portant sur la question nationale ne perdra rien en acuité pour autant? 
L'expression du nationalisme québécois n'est pas nouvelle sur la scène politique canadienne. Sociologues et historiens savent que les termes mêmes de l'Acte de l'Amérique du Nord Britannique de 1867 traduisent le difficile compromis en vertu duquel les Canadiens français acceptaient la formation d'un Canada dominé démographiquement, économiquement et politiquement par les anglo-saxons ${ }^{3}$. Les représentants canadiens-français aux séances de négociations de 1864 entourant une éventuelle confédération n'auraient jamais accepté le regroupement des colonies britanniques d'Amérique du Nord sans l'assurance que les constituantes de la future fédération (les provinces) allaient disposer des juridictions relatives à la préservation de leur spécificité identitaire 4 . En vertu du compromis que scellait la Confédération de 1867, les provinces se voyaient dévolus des pouvoirs relatifs à la culture, à l'éducation et à ce que l'on a appelé plus tard le domaine socials. Ce partage des pouvoirs assurait les provinces d'une certaine maitrise sur la reproduction de leur spécificité culturelle respective.

La configuration un peu étrange de la forme du régime canadien, qui confie au gouvemement fédéral l'essentiel des leviers économiques, en même temps qu'elle accorde aux diverses composantes les instruments de leur reproduction identitaire, soulève depuis lors le problème de la représentation nationale unitaire du Canada. Au-delà de l'évocation du

3 Stanley B. Ryerson, Le Capitalisme et la confédération, Montréal, Parti Pris, 1972.

4 En ce qui conceme les exigences canadiennes-françaises quant à la nécessité d'un palier de gouvemement provincial, voir Jean Cardin et Claude Couture, Histoire du Canada, espace et différences, Québec, Presses de l'université Laval, 1996.

$5 \mathrm{La}$ question du partage des juridictions est clairement présentée dans Manon Tremblay et Marcel R. Pelletier, Le Système parlementaire canadien, Québec, Presses de l'université Laval, 1996. 
«meilleur pays au monde» qui mssène, ou de la représentation de «bon citoyen international» que soulèvent légitimement ses fréquentes participations aux missions de paix de l'ONU, le Canada n'est jamais parvenu à se donner à lui-même la représentation de son unité ni d'une personnalité nationale qui lui serait véritablement propre. Cela d'autant plus que le lancinant particularisme québécois le hante et hi rappelle ce qu'il cherche à refouler : son caractère binational.

Les forces centrifuges qui traversent la dynamique politique canadienne, et dans lesquelles certains ont reconnu une tendance structurelle à la balkanisation?, ne résultent pas que des seules revendications canadiennes-françaises. D'importantes réticences régionalistes s'ajoutent à elles et aggravent encore la tendance à l'éclatement de la fédération. Ainsi par exemple, la dernière élection fédérale a été l'occasion d'une montée en puissance du Reform Party voué à la promotion des intérêts des provinces de l'ouest du Canada. Le mécontentement de ces demières tient au fait que le pays leur semble gouverné en fonction des intérêts du "Canada central». On prend toute la mesure de la tendance à l'éclatement du Canada, si l'on considère que le Reform Party forme actuellement l'opposition officielle et que ce titre avait été détenu, au cours des quatre années précédentes, par le Bloc québécois, un parti souverainiste. Le fait que des partis politiques nationalistes ou régionalistes puissent s'imposer au niveau fédéral traduit assez clairement la difficulté du Canada à produire son unité tant sur le plan politique qu'identitaire. On ne peut

6 Sur les méandres de la production identitaire canadienne, voir Gilles Bourque et Jules Duchastel, L'Identité fragmentée, Montréal, Fides, 1996, de même que Jacques Beauchemin et Victor Armony, "Crise de la représentation de la communauté politique dans le discours constitutionnel canadien", Les Cabiers de recherche sociologique, no. 28, printemps 1997.

7 Gilles Bourque et Anne Legaré, Le Québec, la question nationale, Paris, Maspéro, 1979. 
davantage ignorer le potentiel de division que soulève la question amérindienne. Les premières nations du Canada revendiquent que leur soit concédée l'autonomie gouvernementale sur leurs territoires ancestraux respectifs. Les négociations, plutôt ardues, révèlent une nouvelle fois cette tendance à la balkanisation qui est au cœur de la dynamique canadiennes.

Au Québec, l'expression d'un projet politique visant la souvemineté devait toutefois attendre les années soixante. La Révolution tranquille a été l'occasion d'une politisation du nationalisme québécois au sens où l'objectif tacite qui avait jusque-là consisté pour les Canadiens français à survivre faisait place au projet d'une prise en main du destin de la collectivité. La souveraineté du Québec devint alors l'horizon de bon nombre de Québécois francophones convaincus que la sauvegarde de leur culture en Amérique passait par la réappropriation de tous les leviers de leur développement collectif. Les conflits constitutionnels allèrent donc en s'intensifiant à partir des années soixante. Le Canada anglais se rebiffait alors que le Québec réclamait plus de pouvoir du gouvemement fédéral, en particulier dans les domaines de la culture, de l'éducation et de l'immigration". "What does Quebec want?» se demandait-on au Canada anglais dans les années soixante devant la rigueur des exigences québécoises en matière constitutionnelle ${ }^{10}$. La politisation du vieux fond nationaliste

8 Sur les tensions entre le gouvemement fédéral, le Québec et les nations autochtones, voir Daniel Salée, «Autodétermination autochtone, souveraineté du Québec et fédéralisme canadieny, dans François Rocher [éd.], Bilan québécois du fédéralisme canadien, Montréal, VLB éditeur, 1992.

9 Claude Morin, Les Choses comme elles étaient. Une autobiographie politique, Montréal, Boréal, 1994.

10 «Que veut le Québec ?̀ La question était sur toutes les lèvres au Canada anglais après que le Québec eut rejeté un amendement 
canadien-français devait déboucher sur la tenue du référendum de 1980. En dépit de la défaite des souverainistes, il était évident que cette option politique condensait une masse critique suffisante pour assurer la survie du mouvement, lequel allait s'atteler à préparer «la prochaine fois», ainsi que l'invitait à le faire René Lévesque, au soir de la défaite.

\section{Le référendum de 1995. La redéfinition des enjeux}

Le second référendum portant sur l'accession du Québec à la souveraineté, celui d'octobre 1995, produisit un résultat digne d'un scénario hollywoodien. Les résultats extrêmement serrés ont placé les deux camps littéralement nez à nez et ont contribué à l'exacerbation du conflit ${ }^{11}$. À peine 25000 votes séparèrent les deux options à l'avantage du camp du «non» qui dût se contenter d'un maigre $50,6 \%$ des voix. La conjoncture politique qui s'est ouverte au lendemain du référendum de 1995 a soulevé l'enthousiasme de plusieurs souverainistes qui, en dépit de la défaite, entrevoient que la souveraineté est à portée de la main et que, la prochaine fois, une majorité un peu plus forte de voix obtenues au sein de la seule communauté francophone sera suffisante pour l'emporter. La perspective d'un troisième référendum a immédiatement eu pour effet de radicaliser les

constitutionnel qui, aux yeux du Canada anglais, l'avantageait pourtant. Voir Jean-Louis Roy, Le Choix d'un pays. Le débat constitutionnel Québec-Canada,1960-1976, Montréal, Leméac. 1978.

11 Pierre Drouilly, «Un référendum exemplaire», La Presse, 7 novembre 1995 , p. A-9. La tension est telle que le dévoilement, au mois de juillet 1997, d'une statue commémorant la visite du général de Gaulle au Québec en 1967 a suscité une vive controverse. Plusieurs fédéralistes n'ont pas oublié, en effet, le célèbre "Vive le Québec libre!» du général de Gaulle. 
positions. Le ton s'est durci et on ne cherche plus guère, du côté de certains fédéralistes, à savoir ce que veut le Québec, mais on s'attelle plutôt à mettre au point l'arsenal juridique visant à contrer l'éventuelle accession du Québec à la souveraineté, en même temps que les plus radicaux préparent les lendemains d'une éventuelle proclamation de souverainetét ${ }^{12}$.

L'âpreté du débat s'explique dès lors que l'on considère le positionnement des acteurs sur l'échiquier politique. L'analyse même la plus superficielle de la répartition du vote au demier référendum suffit à montrer le clivage qui s'est creusé entre les tenants de la souveraineté du Québec et les fédéralistes du Québec qui se réclament à la fois d'une identité québécoise et canadienne. Les francophones ont voté en faveur du "oui» dans une proportion d'environ $60 \%$, alors que les anglophones et les

12 Une campagne d'opinion s'organise autour du thème du "partitionnisme». Bon nombre de fédéralistes québécois soutiennent que dans l'éventualité de la souveraineté rien n'empêcherait les collectivités qui le désireraient de demeurer rattachées au Canada. L'ouest de Montréal, où se concentre la majorité des anglophones du Québec, pourrait ainsi réclamer son rattachement au Canada, engendrant de ce fait la partition du territoire québécois. Les souverainistes opposent à ces prétentions les dispositions du droit international portant sur la protection de l'intégrité territoriale. Il est difficile de mesurer la détermination des "partitionnistes", mais il est certain que ce que les souverainistes perçoivent comme une provocation ou bien comme une véritable menace qui envenime considérablement le débat. L'élection de William Johnson, que les anglophones du Québec considèrent eux-mêmes comme un radical, à la tête du regroupement «Alliance Québec» n'est pas de nature à pacifier le débat. Par ailleurs, la Cour Suprême du Canada rendait en août 1998 un jugement statuant qu'une proclamation unilatérale de souveraineté serait inconstitutionnelle. $\mathrm{Ce}$ jugement s'ajoute à l'argumentaire fédéraliste fondé sur la nécessaire primauté du droit. 
allophones ${ }^{13}$ ont voté «non» dans une proportion avoisinant les $95 \%{ }^{14}$. Les allusions au vote ethnique et les références à sl'entrenous" du premier ministre Parizeau au soir de la défaite souverainiste de 1995 expriment l'amertume qui résulte du fait qu'une proportion de $60 \%$ d'appuis francophones n'aura pas suffi à franchir le cap de la victoire face au vote quasi monolithique des minorités du Québec. La majorité, plus importante que jamais, de francophones s'étant prononcés en faveur de la souveraineté ne les pose plus en face de leur étemelle ambivalence, ainsi qu'on l'a tellement répété après la défaite référendaire de 1980 , mais face à ces adversaires que peuvent maintenant constituer à leurs yeux les minorités anglophone et allophone formant un front uni. Le vote massif des minorités du Québec en faveur du "non» a été interprété comme le rejet d'une option politique qui apparait de plus en plus comme un projet exclusivement francophone. Le débat qui nous attend sera donc cette fois d'autant phis difficile qu'il risque de poser face-à-face deux protagonistes qui jusque-là avaient eu la possibilité d'édulcorer l'ampleur de leur opposition. On avait $\mathrm{pu}$, à la suite du référendum de 1980 , secondariser l'effet de blocage engendré par le vote négatif des minorités anglophone et allophones. On ne pouvait, en effet, leur imputer l'entière responsabilité de la défaite du "ouì dans la mesure où les francophones étaient eux-mêmes fortement divisés quant au projet souverainiste. C'est cet ordre des choses qu'est venu rompre le référendum de 1995, comme l'a rappelé brutalement le premier ministre du Québec le soir de la défaite, en soutenant

13 Le comportement électoral des allophones du Québec de même que leur attitude face au projet souverainiste s'apparentent à ceux des anglophones du Québec. C'est la raison pour laquelle le discours souverainiste tend à se les représenter comme un seul et même adversaire.

14 Pierre Drouilly, «Un référendum exemplaire», La Presse, 7 novembre 1995, p. A-9. 
que cette dernière avait été provoquée par «l'argent et le vote ethnique» ${ }^{15}$.

L'éventualité d'une victoire souverainiste à l'occasion d'un prochain référendum alimente donc un débat particulièrement vif. Aux traditionnelles mises en garde des fédéralistes concemant les périls que ferait peser la souveraineté sur le niveau de vie des Québécois, sur le danger de devoir rouvrir l'Accord de libre-échange nord-américain ('ALENA), ou encore sur les conséquences d'une dislocation de l'espace économique canadien $^{16}$, s'ajoutent maintenant les éléments d'une thématique nouvelle portant sur les aspects directement politiques d'une souveraineté québécoise appréhendée ${ }^{17}$. Et il faut reconnaître que le discours anti-souverainiste est parvenu à imposer ces thèmes à telle enseigne que les souverainistes sont acculés à la défensive.

\section{Les nouveaux argumentaires}

On a vù le débat qui conduit «à la prochaine fois» se déployer selon deux arguments étroitement liés. Du côté fédéraliste, on cherche à réactualiser les vieux thèmes de la critique du nationalisme canadien-français d'avant la Révolution tranquille

15 "On a été battus par l'argent et par les votes ethniques !", $\mathrm{La}$ Presse, 2 novembre 1995, p. A-14.

$16 \AA$ À propos des mises en garde fédéralistes au sujet d'une éventuelle souveraineté du Québec, voir Alain G. Gagnon et François Rocher, Répliques aux détracteurs de la souveraineté du Québec, Montréal, VLB éditeur, 1992.

17 Jacques Beauchemin, «Nationalisme québécois et crise du lien socialb, Les Cabiers de recherche sociologique, no 25, automne 1995. 
dont Pierre-Elliot Trudeau s'était fait le champion ${ }^{18}$ : l'ethnicisme et l'antidémocratisme ${ }^{19}$.

La question de lethnicisme. Les critiques qui s'abattent actuellement sur le projet souverainiste soulignent en rouge ses aspects apparemment ethnicistes. Ces allégations se fondent sur un hypothétique exclusivisme dont les sources profondes se trouveraient dans la conception longtemps entretenue dans l'histoire du Québec de la "race» canadienne-française homogène et menacée d'assimilation ${ }^{20}$. À partir de 1840 , en effet, la représentation identitaire canadienne-française est caractérisée par l'obsession de la survivance nationale. L'idée s'impose que la survivance de la race passe par la fidélité à la foi catholique et à l'institution qui la soutient: l'Église. La pierre de touche de la résistance passe nécessairement par une définition culturelle et ethniciste de la collectivité. On cherche à donner corps à ce qu'il y a à défendre et à «substantified» la représentation de la collectivité de telle sorte qu'elle puisse s'apparaître à elle-même comme sujet historique ${ }^{21}$. L'identité canadienne-française se cristallisera autour d'attributs ethnicistes que constitueront l'origine commune, la langue française et la religion catholique. Considérant du haut de l'histoire l'épopée française en Amérique, le chanoine Groulx y reconnut la unaissance d'une races $)^{2}$.

18 Pierre-Elliot Trudeau, , Le Fédéralisme et la société canadiennefrançaise, Montréal, $\mathrm{HMH}, 1967$.

19 Nadia Khouri, Qui a peur de Mordecai Richler?, Montréal, Éditions Balzac, 1995.

20 Esther Délisle, Le Traître et le Juif, Outremont, L'Étincelle éditeur, 1992.

21 Fernand Dumont, Genèse de la société québécoise, Montréal, Boréal, 1993.

22 Lionel Groulx, La Naissance d'une race, Montréal, Bibliothèque de l'Action française, 1919. 
On assista alors à la production de ce que historiens et sociologues québécois ont appellé les mythes fondateurs de la. communauté qui étaient en même temps, comme on l'a dit, des mythes compensatoires ${ }^{23}$. L'imaginaire collectif va en effet se livrer à une véritable réinvention des origines en vertu de laquelle la communauté pourra se représenter sa continuité dans l'histoire. La version dominante du nationalisme canadienfrançais, d'inspiration fortement cléricale, s'est donc d'abord constituée sous la forme d'un conservatisme que Fernand Dumont a saisi sous la belle évocation de «'hiver de la survivance ${ }^{24}$. C'est justement cette définition culturelle et ethniciste de la communauté qui sera brandie, au nom des intérêts de la race, au moment des négociations entourant la Confédération et qui débouchera sur l'exigence des Canadien-. français en faveur de la création de provinces capables de garantir une certaine autonomie.

Les détracteurs du projet souverainiste voudraient aujourd'hui montrer l'actualité de la conception ethniciste et exclusiviste de la collectrivité canadienne-française telle que l'a élaborée l'historiographie du siècle dernier et celle du début du $\mathrm{XXe}$ avec le chanoine Lionel Groulx en particulier. Le nationalisme québécois n'aurait aucune légitimité puisque fondé sur le postulat d'une homogénéité ethnique et appuyé sur un mythe des origines (la Conquête anglaise de 1760) dont l'effet serait d'exclure symboliquement tout individu étranger à cette mémoire collective ${ }^{25}$. Celle-ci se perpétuerait dans la représentation inlassablement ravivée d'un état victimal

23 Sur la réécriture de l'histoire du Canada français et la formation des «mythes compensatoires», on lira Guy Rocher, Le Québec en mutation, Montréal, Hurtubise HMH, 1973 et Serge Gagnon, Le Québec et ses bistoriens de 1840 à 1920, Québec, Les Presses de L'Université Laval, 1978.

24 Fernand Dumont, op. cit., p. 331.

25 Nadia Khouri, op. cit., p. 23. 
originaire. Le nationalisme aurait donc construit une identité collective et un projet d'émancipation dans la réitération douloureuse d'un passé dont il faudrait maintenant chercher à se libérer ${ }^{26}$. Or, une telle représentation identitaire serait, par essence, fermée sur elle-même et donc incapable de reconnaître l'altérité. Le nationalisme fonctionnerait ainsi à l'exclusion, à la célébration ethnique dans la mouvance de laquelle peuvent s'insinuer le racisme et la xénophobie. À cet égard, il ne tient nullement du hasard que surgissent depuis quelques années livres et articles redécouvrant l'œuvre du chanoine Groulx, le plus souvent pour vouer aux gémonies les positions ethnicistes et antisémites de l'historien ${ }^{27}$. Il est toutefois remarquable que la critique du Groulx s'organise très précisément selon la ligne de partage des positions en présence. Du côté souverainiste, on prend ses distances par rapport au clérico-nationalisme fermé à l'altérité pour mieux faire valoir les vertus d'ouverture du néonationalisme issu de la Révolution tranquille ${ }^{28}$. Les adversaires du souverainisme cherchent à recoudre ces deux formes de nationalisme en invoquant avec suspicion le respect dont on continuerait néanmoins d'entourer Groulx.

L'anti-démocratisme. D'autre part, se greffent à cette critique des préoccupations, en pleine recrudescence à l'échelle du monde

26 Cette vue des choses est répandue dans les rangs fédéralistes. À ce sujet, Marc Angenot, Les Idéologies du ressentiment, Montréal, XYZ, 1997.

27 Voir notamment la virulente dénonciation des positions nationalistes et anti-sémites de Groulx dans Esther Delisle, Le Traitre et le Juif, op. cit. On lira aussi avec un peu d'étonnement la réhabiliation de l'oeuvre à laquelle s'adonne Ronald Rudin dans Making History in Twentieth Century Quebec, Toronto, University of Toronto Press, 1997. La lecture la plus nuancée se trouve sans doute chez Femand Dumont, Genèse de la société québécoise, op. cit.

28 Voir Louis Balthazar, Bilan du nationalisme au Québec, Montréal, Hexagone, 1986. 
depuis la chute du mur de Berlin, à propos de la démocratie et des droits de l'homme. Ces préoccupations portant sur la démocratie et la question des droits de l'homme ont pour effet d'alimenter un anti-nationalisme d'autant mieux assuré de luimême que l'histoire vient, semble-t-il, d'enterrer les collectivismes (économiques et politiques) au nom de la tyrannie de la majorité que ceux-ci finimient fatalement par imposer. Ce jugement, inspiré par l'un des retoumements politiques les plus importants du XXe siècle, place les nationalistes sur la défensive et les accule à justifier le caractère apparemment antidémocratique et à contre-courant de l'histoire de leur projet politique ${ }^{29}$. En cela, certains fédéralistes qui, aujourd'hui, s'échinent en leçons de démocratie, saisissent, en quelque sorte, la balle au bond. Cela ne signifie aucunement que la résurgence de ces préoccupations soit artificielle. Les questions du droit, de la démocratie et de la tolérance s'imposent au débat de manière incontoumable, tant il est vrai que le destin des sociétés contemporaines se jouera dans le cadre d'un pluralisme qu'il s'agira d'aménager politiquement ${ }^{30}$. La polarisation du débat politique entre droits individuels et collectifs relève en effet directement de ces préoccupations envers la démocratie et les droits des minorités ${ }^{31}$. Voilà qui ouvre la

29 Voir notamment Alain Touraine, Poumons-nous vive ensemble?, Paris, Fayard, 1997 et Alain Minc, La Vengeance des nations, Paris, Librairie générale française, 1993.

30 Ce n'est nullement un hasard si, de part et d'autre de l'Atlantique, surgissent d'importantes préoccupations portant sur le multiculturalisme et la question du pluralisme. Voir entre autres Michel Wieviorka, Une société fragmentée? Le multiculturalisme en débat, Paris, La Découverte. 1996; Charles Taylor, Multiculturalisme, différence et démocratie, Paris, Aubier, 1994; et Amy Gutman [éd.], Multiculturalism, Princeton, Princeton University Press, 1994.

31 James Tully, Strange multiplicity. Constitutionalism in an age of diversity, Cambridge, Cambrige University Press. 1995 et Charles Taylor, Rapprocher les solitudes. Écrits sur le fédéralisme et le nationalisme au Canada, Québec, Presses de l'université Laval, 1992. 
voie à la formulation de nouvelles exigences démocratiques auxquelles les souverainistes sont invités à se conformer. Au Québec, ces préoccupations sont toutes entières investies dans la question des droits des minorités et de la légitimité de l'imposition d'une future souveraineté qui ne représenterait, à toutes fins pratiques, que les intérêts d'une majorité de francophones. Les souverainistes soutiennent que les droits collectifs ne sont nullement incompatibles avec les droits individuels et que, plus précisément, ces derniers ne signifient rien sans la nécessaire référence à la réalité socio-historique qui pose le citoyen porteur de droits sur l'horizon d'une identité collective. Livres et articles savants se multiplient depuis quelques années, qui cherchent à arrimer ces deux univers de droits apparemment inconciliables ${ }^{32}$. Les souverainistes prétendent ainsi que le nationalisme dont ils se réclament est parfaitement ouvert au pluralisme et mû par une volonté d'inclusion des minorités dans l'ensemble national. On se propose alors de promouvoir l'existence d'une communauté majoritaire formée par l'histoire sans compromettre les droits des groupes qui ne partagent pas ce même rapport à l'histoire du Québec. Dans ce contexte, la revendication du français comme langue publique commune ne serait pas antidémocratique mais constituerait, au contraire, une assise de la démocratie en ce qu'elle foumirait aux citoyens de toutes provenances un moyen d'insertion dans l'espace public (pluraliste) au sein duquel chacun pourrait faire valoir ses intérêts ${ }^{33}$. Entrainés à leur corps défendant dans le jeu de cette opposition binaire entre droits

32 On retrouve des tentatives de conciliation de ces termes apparemment opposé dans Guy Laforest, De la prudence, Montréal, Boréal. 1993 et dans Will Kymlicka, Multicultural citizenship, New York, Oxford Universiy Press. 1995.

33 Cette position est clairement définie dans Gérard Bouchard, «La Nation au singulier et au pluriel. L'avenir de la culture nationale comme "paradigme» de la société québécoise», Les Cabiers de recherche sociologique, no 25 , automne 1995, pp.79-97. 
individuels et collectifs, Québécois francophones et Québécois d'autres origines, exclusion et inclusion, les souverainistes voient émerger dans leur propres rangs des positions divergentes quant à l'interprétation de ces oppositions. Gérard Bouchard critique alors, au nom de l'ethnicisme qu'elle véhicule tacitement, la position de Femand Dumont qui pose quant à lui l'existence d'une nation canadienne-française (et non québécoise) de laquelle émergerait en réalité le projet souverainiste ${ }^{34}$. Du côté fédéraliste, on rejette les prétentions souverainistes en faveur de l'ouverture et du respect des minorités en s'en tenant fermement à la conception étroitement libérale de la citoyenneté pour laquelle n'existent que des "patriotismes constitutionnels» au sens où l'entend Habermas. C'est dans cette perspective que le premier ministre du Canada estimait aux lendemains du demier référendum que la majorité simple $(50 \%$ plus une voix) ne suffirait pas à assurer la légitimité d'un prochain vote référendaire si celui-ci devait signifier la victoire des forces souverainistes ${ }^{35}$.

\section{Le lieu d'un problème}

La position adoptée par le premier ministre canadien alimente un faux débat en même temps qu'elle soulève un vrai problème. La question du seuil à partir duquel un vote majoritaire en faveur de la souveraineté pourmit être légitime s'inscrit dans un faux débat dans la mesure où la remise en cause de la valeur de la majorité simple correspond en réalité à une

34 Gérard Bouchard, "Contre le retour d'une pensée ethnique», allocution prononcée dans le cadre du collloque de I'Association canadienne-française pour l'avancement des sciences, à Québec le 12 mai 1998.

35 «Une majorité simple ne suffirait pas», La Presse, 26 mai 1997, p. 1. 
stratégie fédéraliste de blocage. Par ailleurs, la question de la légitimité d'un vote serré soulève un véritable problème. Une courte victoire du «oui» à un éventuel troisième référendum soulèverait d'abord un important problème de faisabilité. Non pas parce que l'on vient de découvrir du côté fédéraliste que la majorité simple est insuffisante à une déclaration d'indépendance politique, mais pour des raisons de nature essentiellement politique. Ces problèmes de faisabilité renvoient d'abord au fait qu'une victoire du «oui» serait forcément très mince. Il est en effet douteux que l'on parvienne à accroître très sensiblement la proportion de francophones favorables à la souveraineté au-delà des $60 \%$ actuels. La position de la majorité francophone qui aurait alors voté en faveur de la souveraineté serait non seulement inconfortable, mais cette victoire précaire risquerait même de fragiliser sa détermination, certains préférant peut-être, dans les circonstances, lancer une nouvelle perche au Canada dans la perspective d'ultimes négociations. On peut penser qu'une certaine mauvaise conscience francophone viendrait miner de l'intérieur même des forces souverainistes la proclamation sans complexe de la souveraineté. Bref, les souverainistes eux-mêmes risquent d'être divisés face à la pertinence d'une déclaration de souveraineté à la suite d'une victoire peu convaincante. Sur le plan idéologique, une mince majorité pourra toujours être invoquée par les perdants comme une quasi-victoire de fait. On n'a qu'à se rappeler les incantations souverainistes autour du $49 \%$ de uoui» qui visaient à fragiliser la légitimité d'un «non» obtenu à l'arraché. L'inverse ne manquera pas de se produire. Il est illusoire de penser qu'une offensive idéologique visant à discréditer une victoire arrachée par la peau des dents n'aurait pas d'effet chez les souverainistes aux ferveurs plus volatiles qui auront voté oui par inclination «naturelle» phutôt que par convictions.

De manière générale, la question du caractère multinational et pluriethnique du Québec est trop facilement éludée dans le discours souverainiste. Pourtant, cette pluralité est non 
seulement une réalité sociologique, mais elle constitue la réalité politique du Québec. La victoire d'un oui signifierait celle d'une majorité de francophones sur les minorités anglophone et allophones. Il est facile d'imaginer la création de toutes sortes de coalition de circonstances à partir desquelles on tenterait de délégitimer l'accession à la souveraineté. Il est certain que l'on verrait les sensibilités (nationale et ethniques) s'exprimer très vivement. De la même manière que les souverainistes répètent, à bon droit, que la souveraineté du Québec constitue une question politique et non juridique, on ne pourrait discréditer les protestations des communautés minoritaires sous prétexte du nouveau cadre juridique résultant d'une souveraineté advenue. Un Québec souverain verrait surgir en son sein une question nationale anglo-canadienne avec laquelle 1 deva apprendre à vivre. Le partitionnisme des anglophones de l'ouest de Montréal constitue la réplique anticipée à cette prétendue dénégation de droits démocratiques. Cette manœuvre est dénoncée au nom de son opportunisme, mais il n'en demeure pas moins que les souverainistes ont tendance à sous-estimer les problèmes liés à la légitimité de leur projet. Le Québec dont ils rêvent est dores et déjà une société multinationale et pluriethnique. Cette réalité socio-politique, qu'ils le veuillent ou non, soulève la question de la légitimité de leur entreprise. La victoire d'un "oui» au prochain référendum signifierait bel et bien celle d'une majorité de francophones sur les minorités anglophone et allophones. Non seulement la souveraineté devrait-elle défendre sa légitimité dans un contexte où toute une composante de la société s'y serait opposée, mais, plus grave encore, elle se heurterait, on l'a évoqué plus haut, à un important problème de faisabilité du strict point de vue politique. Cela ne veut pas dire qu'une proclamation de souveraineté consécutive à une victoire référendaire serait nécessairement illégitime, mais un tel clivage signale à tout le moins les difficultés auxquelles se heurterait le nationalisme francophone quant à la mise en ceuvre concrète d'une éventuelle souveraineté. 


\section{La souveraineté.}

\section{Espoirs et empêchements}

Au moment où triomphait la Révolution tranquille, au cours des années soixante, la sociologie québécoise s'interrogeait à propos des blocages et des recommencements qui avaient prétendument empêché, jusque-là, l'avénement de la modemité au Québec ${ }^{36}$. Sur le plan sociologique, la représentation du «retard du Québec» s'est alors traduite dans cette thèse selon laquelle le développement des idéologies, prisonnier du traditionalisme, ne se serait pas accordé au mouvement de l'industrialisation ${ }^{37}$. La société québécoise, jusqu'à la Révolution tranquille, aurait été captive d'un «empêchement», comme l'a prétendu Fernand Dumont ${ }^{38}$, qui lui aurait en quelque sorte interdit la possibilité d'advenir à la modemité et d'ouvrir devant elle les chemins de son émancipation politique. Plus de vingt ans plus tard, Dumont précisa cette thèse en posant, dans une perspective généalogique, que les sédiments de la référence identitaire canadienne-française élaborée au XIXe siècle sont toujours secrètement actifs dans la conscience québécoise contemporaine $^{39}$. La société québécoise aurait peine à échapper à sa définition d'elle-même, celle qu'a produite un XIXe siècle marqué par la domination et la peur de l'assimilation. Les effets permanents de cette référence identitaire feraient en sorte que subsistent dans l'inconscient collectif de vieux complexes (ceux des peuples colonisés) et d'anciennes nostalgies (l'«abandon» de

36 Marcel Rioux, «Sur l'évolution des idéologies au Québeo, Bruxelles, Revue de l'institut de sociologie, 1968.

37 Ibid., p. 112.

38 Fernand Dumont, La Vigile du Québec, Montréal, Hurtubise HMH, 1971.

39 Fernand Dumont, Genèse de la société québécoise, op. cit., p. 331. 
la France) qui s'opposeraient à l'idée de la souveraineté du Québec comme autant de fantômes menaçants ${ }^{40}$.

Quoi qu'il en soit de la validité de ces thèses, empreintes d'un culturalisme peut-être un peu trop insensible aux transformations concrètes de la régulation des rapports sociaux qui s'opéraient dans le Québec des années soixante ${ }^{41}$, il est certain que le Québec souverainiste francophone d'aujourd'hui est en pleine effervescence, fort des espoirs que lui ouvrent les résultats du dernier référendum, mais aussi "captif» de nouveaux "empêchements». Ceux-là ne sont pas enfouis au fond de sa vielle référence identitaire ainsi que l'a supposé Fernand Dumont, mais se trouvent au cœur de la réalité socio-politique concrète du Québec contemporain. La sagesse impose de les inventorier sans ne rien cacher des difficultés à venir, sans non plus renoncer, comme par avance, au projet lui-même au nom de ces mêmes difficultés.

Le danger qui guette les Québécois de toutes origines est celui d'un infléchissement du débat sur la souveraineté en vertu duquel celui-ci n'apparaitrait plus que comme le seul projet des francophones, projet qu'il ne resterait plus qu'à imposer aux

$40 \mathrm{La}$ thèse d'un traumatisme original est largement reprise dans l'historiographie et la sociographie québécoise. Voir, par exemple, Christian Dufour, Le Défi québécois, Montréal, L'Hexagone, 1989.

41 Cet hypothétique passage du Québec à la modemité au cours de la Révolution tranquille correspond plutôt au fait que le Québec développait alors «son» État-providence. Le néo-nationalisme, qui naissait dans cette conjoncture, alimentait le projet politique de l'État-providence dans la mesure où ce dernier fondait sa légitimité sur la nécessité d'un progrès de la collectivité (le progrès social), en même temps que ce néo-nationalisme se trouvait renforcé par les réussites sociales inspirées des politiques providentialistes. On a pu, dans ce contexte, produire la représentation nouvelle d'une collectivité francophone capable et volontaire. Voir à ce sujet Louis Balthazar, op. cit. 
minorités en augmentant le pourcentage du vote souverainiste au sein de la seule communauté francophone. Plusieurs francophones estiment qu'on ne peut esquiver la vérité sociologique qu'exprime la quasi unanimité de l'appui des communautés anglophone et allophones au «non». C'est ce sombre diagnostic que semblaient formuler les propos du premier ministre Parizeau, au soir de la défaite en octobre 1995, lorsqu'il rappelait que les "trois cinquièmes de ce que nous sommes ont voté oui» et qu'on va se donner «un pays à nous», "gens aussi bien de droite que de gauche, entre les syndicalistes

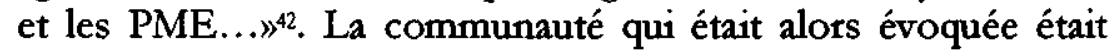
bien celle de la majorité francophone que le discours de monsieur Parizeau posait alors comme politiquement autosuffisante ${ }^{43}$. Cette position est dangereuse parce qu'elle divise plus encore la société québécoise et n'augure rien d'encourageant pour la réconciliation à laquelle devra de toute façon s'atteler un éventuel Québec souverain.

Le risque de voir «s'ethnicisen la question nationale devrait faire réfléchir quant à l'opportunité de la tenue d'un prochain référendum. Il paraît souhaitable que les aspirations nationalistes francophones puissent être satisfaites dans le cadre d'un réaménagement du fédéralisme canadien. Sans entrer dans le détail de ce que devrait concrètement réunir ce réamanénagement pour qu'il soit acceptable, il semble évident que seul la reconnaissance franche et sans détour de la réalité

42 "On a été battus par l'argent et par les votes ethniques!», $L a$ Presse, 2 novembre 1995, p. A-14.

43 Le premier ministre Parizeau a toujours insisté sur le fait qu'il ne désignait pas ainsi le groupe ethnique que constituerait l'ensemble des Canadiens français, mais plutôt l'ensemble des francophones de toutes origines du Québec. Aux yeux de monsieur Parizeau, l'adversaire politique est donc constitué de ce qui s'oppose au nationalisme francophone et non pas de regroupements ethniques particuliers. 
nationale québécoise est susceptible de satisfaire ceux qui aujourd'hui ne voient d'autres solutions que la souveraineté pure et simple. Cela implique naturellement des gestes d'ouverture de la part du reste du Canada dont on ne voit malheureusement guère les manifestation depuis la tenue du référendum de 1995 . Il résulte de ce blocage que les nationalistes québécois se trouveront bientôt "acculés» à relancer le processus menant à la souveraineté alors même que plusieurs, dont l'auteur de ces lignes, entrevoient les dangers que recèlent sa proclamation dans un contexte d'extrême division et que plusieurs pressentent que le contentieux pourraient être dénoué par voie constitutionelle pour peu que soit reconnue un certain nombre d'exigences liées à la pérennité de la culture francophone au Québec.

Si l'embâcle politique persiste au Canada et que la tenue d'un référendum dont plusieurs subodorent déjà les périls devait s'imposer, le problème politique qui se poserait aux francophones consisterait à savoir s'ils veulent d'un Québec souverain qu'ils seraient parvenus à imposer par la force du nombre à des minorités largement réfractaires à ce projet politique. Les conditions d'un douloureux face-à-face sont posées et appellent plus que jamais un esprit de tolérance et d'ouverture, celui qui a toujours caractérisé le nationalisme québécois depuis la Révolution tranquille. Du point de vue des éventuels "perdants» toutefois, rien ne devrait faire oublier que si le projet souverainiste origine bien évidemment de la communauté nationale que forment les Québécois francophones, rien n'autorise à prétendre que le projet soit en lui-même discriminatoire et négateur des droits des minorités. Le projet souverainiste, dans son expression la plus commune, celle qui se retrouve dans le projet du Parti québécois, répond à toutes les exigences d'une démarche démocratique respectueuse des droits de chacun et ouverte à l'altérité. 
Dans l'un de ces demiers ouvrages, Dumont appelait les Québécois à joindre à leur opiniâtre volonté de survivance d'antan le "courage de la liberté» qu'appelle la conjoncture actuelle ${ }^{44}$. Cet appel, les Québécois, s'ils veulent l'entendre, fera en sorte qu'ils devront assumer clairement les difficultés associées à une souveraineté qu'ils auront peine à faire valoir auprès de leurs concitoyens anglophones et allophones. Les Québécois, s'ils votent majoritairement en faveur de la souveraineté lors d'un prochain référendum, auront pris une décision difficile. La société québécoise se sem donnée les moyens de son autonomie politique, mais la conquête de cette autonomie sera plus longue et plus difficile que ne le laisse entendre le discours souveministe. $\mathrm{La}$ reconnaissance internationale d'un Québec souverain ne posera sans doute pas de problèmes, mais on ne peut pas en dire autant, par exemple, du partage des actifs et du passif canadien. De même, il n'est pas difficile d'imaginer que certains éléments plus radicaux des minorités qui se sentiront floués au lendemain d'une victoire, à leurs yeux peu convaincante, s'activeront à entraver les négociations que le Québec entreprendra avec son nouveau partenaire canadien. Le débat que doivent continuer de mener les Québécois entre eux doit se dérouler à l'abri du catastrophisme fédéraliste tout autant que de la célébration prématurée de l'accession sans soubresauts à la souveraineté nationale qui en constitue l'envers optimiste.

La seule véritable question qui se pose alors est de savoir si le gouvernement du Québec voudra ou non proclamer la souveraineté, fondée sur une courte victoire référendaire, en assumant les conséquences politiques et sociales d'une décision qui sera de toute façon tenue pour illégitime par plusieurs, peu importe le seuil de la majorité qui aura été retenu.

44 Fernand Dumont, Genèse de la société québécoise, op. cit., p. 336. 